\title{
AGRICULTURA FAMILIAR E TECNOLOGIAS DE INFORMAÇÃO E COMUNICAÇÃO (TICs): PROJETO PILOTO VALE DO CAÍ
}

\author{
Luana de Souza Barcelos ${ }^{1}$ \\ Grébori Anderson Silva ${ }^{2}$ \\ Douglas Ritt ${ }^{3}$ \\ Angélica Lubaczwski ${ }^{4}$ \\ Cidonea Machado Deponti ${ }^{5}$
}

\section{RESUMO}

O desenvolvimento de técnicas de gestão que contemplem as particularidades da agricultura familiar e as formas pelas quais ela pode se inserir de forma competitiva e sustentada no mercado são escassas e mal adaptadas. $\mathrm{O}$ presente artigo é resultado do projeto de pesquisa intitulado Desenvolvimento Rural e Tecnologias de Informação e Comunicação (TICs), que objetiva analisar as possibilidades de introdução de TICs para a agricultura familiar no Vale do Caí, com a experiência de extensão piloto em Montenegro-RS, município polo desta região, visando o desenvolvimento rural e regional. $\mathrm{O}$ artigo foca-se nas atividades de extensão que apresentam como objetivo principal a capacitação das famílias de agricultores familiares em TICs a partir de diálogos, de discussões, de negociação e da construção conjunta do conhecimento. Para tanto, foram realizadas oficinas de integração com os agricultores versando sobre a utilização das principais TICs e, posteriormente, será construído de forma conjunta planilhas eletrônicas para a gestão da propriedade rural. Além disso, ocorre o monitoramento in loco para acompanhamento e para auxílio ao processo de registro das informações visando construir uma metodologia coletiva com os agricultores, de forma que possam tornar-se autônomos nos processos gerenciais.

Palavras-chave: Agricultura familiar. Tecnologias de informação e comunicação. Desenvolvimento rural e regional. Gestão da propriedade rural.

\begin{abstract}
The development of management techniques that address the particularities of family farming and the ways in which it can insert itself competitively and sustainably in the market are scarce and poorly fitted. This article is the result of a research project entitled Rural Development and Information and Communication Technologies (ICTs), which aims to analyze the possibilities of introducing ICTs to the family farm in the Caí Valley, with the experience of pilot extension in Montenegro-RS, the main city of the region, aimed at rural and regional development. The article focuses on the extension activities that have as main objective the training of family farmers in ICT from dialogues, discussion, negotiation and

\footnotetext{
${ }^{1}$ Aluna do Curso de Administração da Universidade de Santa Cruz do Sul - UNISC. <barcelos.luana@yahoo.com.br>

${ }^{2}$ Aluno do Curso de Administração da Universidade de Santa Cruz do Sul - UNISC. <greborisilva@agrogen.com.br>
Aluno do Curso de Administração da Universidade de Santa Cruz do Sul - UNISC. <douglasritt@hotmail.com>

${ }^{4}$ Aluna do Curso de Administração da Universidade de Santa Cruz do Sul - UNISC. <angélica_luba@yahoo.com.br>

${ }^{5}$ Professora do Programa de Pós-Graduação Mestrado e Doutorado em Desenvolvimento Regional da Universidade de Santa Cruz do Sul - UNISC < $\underline{\text { cidonea@unisc.br> }}$
} 
joint construction of knowledge. To this end, integration workshops dealing with farmers on the use of the main ICT and subsequently will be built set of spreadsheets to manage the farm were performed. In addition, monitoring in place to monitor and aid the process of recording information is aiming to build a collective approach with farmers, so they can become selfemployed in managerial processes.

Keywords: Family agriculture. Information and communication technologies. Rural and regional development. Farm management technologies.

\section{INTRODUÇÃO}

A visão que associava o espaço rural unicamente como local de produção agrícola cada vez mais perde poder explicativo na medida em que se agregam novas funções para esse espaço. O desenvolvimento rural, conforme sustenta Ploeg et al. (2002), é sobre a reconstrução de novas redes, a revalorização e a recombinação dos recursos, a coordenação e a remolduração do social e do material e ainda a renovação do capital social, cultural e ecológico.

Nesse contexto ampliado de desenvolvimento rural, este meio não deveria ser visto como um local distante e atrasado pelos que vivem no meio urbano e industrial, mas sim como um ícone de diversidade que está em constante desenvolvimento. As Tecnologias de Informação e Comunicação (TICs), especialmente a internet, tornaram-se uma necessidade para o meio rural, tanto quanto já era para o urbano, visando a atender à demanda por conhecimento e informações atualizadas e constantes (VIEIRO; e SILVEIRA, 2011). Segundo Thornton (2003), a internet é uma ferramenta fundamental para o acesso ao conhecimento, porém é crucial pensar no processo de apropriação individual dessa tecnologia, pois a adoção das TICs inclui fatores como o acesso e o uso. Nesse sentido, o autor destaca que é essencial que as informações disponíveis levem em consideração os saberes locais e que os agricultores não sejam vistos como meros receptores, mas também como parte atuante dos processos interativos de comunicação e de informação. A adoção da internet somente será efetiva quando o produtor tiver incorporado seu uso na rotina cotidiana, ou seja, quando a empregar com naturalidade na busca por soluções ou alternativas aos problemas do seu dia a dia.

Para compreensão deste processo utilizou-se a metodologia qualitativa, primeiramente sendo realizadas entrevistas semiestruturadas para traçar o perfil dos 10 agricultores componentes do Projeto Piloto e compreender o uso das TICs. Para tanto, são realizadas oficinas de integração sobre o acesso aos computadores e as principais tecnologias de informação e de comunicação e visitas quinzenais de acompanhamento. Posteriormente 
serão elaboradas planilhas eletrônicas $(E x c e l)$ de forma conjunta com os agricultores para introdução de informações sobre o estabelecimento rural e, assim, possibilitar o acompanhamento das principais variáveis econômicas e o andamento da propriedade, colaborando para a tomada de decisão.

O presente artigo, além desta introdução, apresenta na primeira seção uma discussão sobre o papel das TICs para o desenvolvimento rural. Na segunda parte apresenta-se a metodologia da pesquisa e na terceira discute-se o uso das TICs pela agricultura familiar, contextualizando o caso do Vale do Caí. A última parte refere-se à análise dos resultados da pesquisa e, por fim, registra as considerações finais.

\section{PAPEL DAS TICS NO DESENVOLVIMENTO RURAL}

O desenvolvimento rural também está relacionado com a reconfiguração dos recursos rurais: terra, trabalho, natureza, animais, plantas, redes, mercados, relações entre rural e urbano, todos devendo ser reformados e recombinados. O novo paradigma de desenvolvimento rural deve ajudar a clarear como novos recursos-base são criados, como os irrelevantes tornam-se valorizados e como combiná-los com outros recursos. As TICs podem reforçar o desenvolvimento rural e colaborar para a diversificação de atividades tradicionais das zonas rurais, como a agricultura, a silvicultura, a pesca, a exploração mineira, o setor da madeira, o artesanato e o turismo e contribuir com informações meteorológicas, dados econômicos, preços a praticar e informações sobre a concorrência, possibilitando acesso a novos mercados. As redes de informática reduzem os custos de transação, fornecendo a flexibilidade para agir rapidamente face às novas situações comerciais (MILLARD, 2000).

Todo indivíduo possui o direito de informação e as TICs contribuem para o desenvolvimento destas. Porém, conforme os dados coletados por Schwartz (2007), a maior parte da população no Brasil, em 2005, não possuía acesso a Internet. Apenas, cerca de 14\% dos habitantes do país possuía acesso à tecnologia em suas residências. A maioria dos internautas era adolescente, em torno de $34 \%$. Conforme a idade aumentava, o percentual diminuía, atingindo 3,3\% para as pessoas com mais de 60 anos de idade. Outro fator relevante é que indivíduos com mais de 15 anos de estudos são os que mais utilizam a rede. Os trabalhadores rurais são os que menos possuem acesso às redes de comunicação, chegando atingir apenas $1,7 \%$.

A mesma autora supracitada, Schwartz, em 2012, realizou pesquisa de doutorado e observou que os moradores do campo que, tradicionalmente, apresentavam-se isolados pela distância das cidades e pela precariedade das estradas, começaram a utilizar telefones 
celulares. Em 2010, “os lares da zona rural apresentaram o maior crescimento de posse de telefone móvel, passando de 58 por cento em 2009 para 68 por cento em 2010" (COMITÊ GESTOR DA INTERNET NO BRASIL, 2011 apud Schwartz (2012, p. 13).

Outro aliado às tecnologias de informação e comunicação são os telefones móveis e fixos. Ainda de acordo com a pesquisa de Schwartz (2007), os telefones fixos, em 2006, obtiveram uma baixa de 42,1 para 41,9 milhões. Sendo assim, para cada grupo de 100 habitantes há 22,4 telefones. Isso ocorreu devido ao telefone único, ou seja, o mesmo aparelho funciona como fixo we móvel, dependendo do tipo de ligação realizada. Ainda é possível hoje realizar ligações telefônicas pela internet através de programas como o Skype.

Para Schwartz (2007), atualmente, a tecnologia mais utilizada é a telefonia móvel e cerca de 100 milhões de aparelhos foram habilitados. Se comparar-se o acesso à rede virtual entre as pessoas com pouca ou nenhuma instrução ver-se-á que elas possuem cinco vezes mais o acesso ao celular e que as de baixa renda utilizam o telefone três vezes mais do que o acesso a Internet. Além disso, a área rural é a que menos possui acesso ao telefone como a utilização da rede virtual.

Bordenave (1983, p. 9) destaca que no meio rural a comunicação é muito importante - "o desenvolvimento rural gira ao redor da comunicação, visto que os agricultores necessitam dela para tomar decisões de produção e de convivência". O autor acredita que a comunidade rural sofre com a falta de comunicação, estando relacionada com o analfabetismo, o baixo nível de instrução, o trabalho pesado durante várias horas do dia e causando, assim, o desejo de descansar e de dormir do que sair de casa.

Thornton (2003) reforça que, atualmente, para que a internet chegue até o meio rural, há algumas barreiras como a idade, o estilo de vida, a situação econômica, a família, a rotina de trabalho e o nível educacional, fatores que irão interferir na implantação de uma rede. Para Vieiro e Souza (2008), a base estratégica das nações que mais se desenvolveram no século $\mathrm{XX}$ foi a capacidade de gerar e de utilizar as novas tecnologias de informação e de comunicação (TICs) na agricultura. Através do investimento em pesquisa e em seu uso na agricultura, uma nação é capaz de avançar através de seus próprios meios e estruturas, sem depender da aquisição, mediante pagamento, de informações, de processos e de produtos gerados em outros locais (RIPPER FILHO, 1994 apud VIEIRO e SOUZA, 2008 p. 2).

Com base nos autores supracitados, pode-se inferir que o desenvolvimento rural apresenta inter-relações com as tecnologias de informação e de comunicação, principalmente quando se considera questões relativas à produção do conhecimento, aos processos de ensinoaprendizagem, à gestão da propriedade rural, à comunicação no campo e à inclusão digital. 


\section{METODOLOGIA}

A metodologia utilizada é a qualitativa, realizada a partir da seleção de 10 famílias que compõem o Projeto Piloto. Estas famílias foram selecionadas pelos parceiros do Projeto: EMATER/RS-ASCAR (Empresa de Assistência Técnica e Extensão Rural do Rio Grande do Sul) e Sindicato dos Trabalhadores Rurais de Montenegro-RS. O critério de seleção baseou-se no acesso ao computador e à internet, não sendo uma amostra probabilística, pois se trata de um piloto. As atividades do projeto dividiram-se em duas partes: uma voltada para pesquisa e outra voltada para extensão. No que se refere às atividades de pesquisa, primeiramente foram realizadas entrevistas semiestruturadas com as dez famílias componentes do projeto Piloto, objetivando verificar o perfil das famílias, o acesso e o uso de TICs pelos agricultores familiares.

Dentre as atividades de extensão tecnológica destacam-se: a) oficinas de integração já foram realizadas quatro oficinas, nos meses de agosto e outubro de 2013 e de abril e maio de 2014, na qual os agricultores tiveram acesso aos computadores e as principais tecnologias de informação e de comunicação, tais como o uso da internet, e-mail, acesso a sites, comunicadores instantâneos. Nas oficinas também foram trabalhadas questões referentes ao registro das informações nas planilhas eletrônicas e à importância do controle de despesas e de receitas; b) realização de uma palestra por um dos professores componentes da equipe do projeto sobre a importância da educação financeira e o controle dos gastos; c) acompanhamento dos agricultores in loco uma vez por mês para o monitoramento e para auxílio ao processo de registro das informações realizado pelos bolsistas e integrantes do projeto, d) Construção coletiva de planilhas eletrônicas (Excel) de com os agricultores para introdução de informações sobre o estabelecimento rural e, assim, possibilitar o acompanhamento das principais variáveis econômicas e o andamento da propriedade, colaborando para a tomada de decisão. Nesta fase, os agricultores que apresentarem maior domínio sobre o uso de tais tecnologias colaboram na introdução deste hábito nas famílias dos demais agricultores participantes do projeto. No entanto, o domínio da tecnologia, o controle e a gestão do estabelecimento rural dependem da escolaridade, da capacitação, do conhecimento e da adaptação à inovação dos agricultores familiares.

\section{O USO DE TICs PELA AGRICULTURA FAMILIAR}

O desenvolvimento de novas tecnologias oriundas das de informação, aliado à complexidade e ao dinamismo dos mercados atuais, impacta a gestão rural e suas decisões 
estratégicas, levando ao remodelamento das rotinas e à resolução de problemas até então tidos como insolúveis pelos agricultores (MARION; SEGATTI, 2006).

Ao se tratar de tecnologias para a agricultura familiar, Batalha, Buainain e Souza Filho (2004, p. 1) destacam que "grande parte deste esforço está sendo dedicado ao desenvolvimento e difusão de tecnologias de processo, de materiais e de produtos e serviços". No entanto, os esforços voltados para as tecnologias de gestão e de informação são ainda incipientes. Os autores argumentam que as tecnologias de gestão são fundamentais para a competitividade da agricultura familiar brasileira. "As tecnologias para a gestão da agricultura familiar são parte fundamental dos conhecimentos e das técnicas de uma empresa rural necessita para a obtenção do sucesso do empreendimento" (BATALHA; BUAINAIN; SOUZA FILHO, 2004, p. 1). Contudo, é preciso reconhecer que muito pouco tem sido feito em termos de desenvolvimento de técnicas de gestão que contemplem as particularidades da agricultura familiar e as formas pelas quais ela pode inserir-se de forma competitiva e sustentada no mercado.

De acordo com Buainain, Souza e Silveira (2002), com a difusão dessas tecnologias pode haver o aumento ou diminuição das vantagens dos agricultores familiares. Qualquer tipo de sistema de gerenciamento, mesmo que seja de fácil acesso, apresenta dificuldades de implantação. O fato de não possuírem a cultura da elaboração de registros escritos, comuns entre os agricultores, obstaculiza a utilização de práticas gerenciais, levando a uma situação altamente insatisfatória no que se refere a técnicas de gestão, o que pode comprometer, segundo os autores, a sustentabilidade e a competitividade dos empreendimentos.

Batalha, Buainain e Souza Filho (2004) ainda destacam outros fatores que não privilegiam os aspectos de gestão, tais como: a pouca cultura (formal e informal) do agricultor neste assunto, o baixo nível de qualificação dos técnicos extensionistas em tecnologias de gestão, a inadequação das ferramentas disponíveis na literatura, a descapitalização dos agricultores (que impede a contratação de técnicos efetivamente qualificados no assunto) e as políticas públicas de estímulo ao setor.

A maioria dos agricultores familiares, normalmente não tem possibilidade financeira de contratar um profissional administrativo. Faz-se necessário, portanto, a elaboração de um sistema de fácil aplicação e manuseio, além de flexível para calcular o custeio de qualquer produto ou criação explorados na propriedade (BATALHA et al., 2005).

Dessa forma, verificam-se diversas dificuldades para a introdução e a utilização de TICs pela agricultura familiar. A disponibilização de técnicas gerenciais apropriadas para a 
agricultura familiar visa a contribuir para a promoção da sustentabilidade econômica desses estabelecimentos rurais.

\subsection{O uso de TICs no Vale do Caí: Projeto Piloto Montenegro-RS}

A região do Vale do Caí ocupa uma área de $1.854 \mathrm{~km}^{2}$, o que corresponde a $0,65 \%$ da área total do estado. Localiza-se nas regiões fisiográficas da Depressão Central e parte da Encosta da Serra. Compreende 19 municípios: Alto Feliz, Barão, Bom Princípio, Brochier, Capela de Santana, Feliz, Harmonia, Linha Nova, Maratá, Montenegro, Pareci Novo, Salvador do Sul, São José do Hortêncio, São José do Sul, São Pedro da Serra, São Sebastião do Caí, São Vendelino, Tupandi e Vale Real. O Vale do Caí encontra-se próximo da região metropolitana de Porto Alegre e a principal rodovia que liga o Vale à capital do estado é a BR - 386, chamada Estrada da Produção (FEE, 2013).

O Vale do Caí apresenta 170.659 habitantes e uma densidade demográfica de 92,0 hab $/ \mathrm{km}^{2}$, dispondo de um PIB pm de $\mathrm{R} \$$ 3.790.443/ano e um PIB per capita de $\mathrm{R} \$ 22.348,00$ (FEE, 2013). As exportações totais alcançaram no ano de 2010 um montante de U\$ FOB 944.294.865. O Índice de Desenvolvimento Humano - IDH do Vale do Caí tem uma média de 0,817, enquanto que a do estado é de 0,814 (FEE, 2006).

Conforme Souza e Binkowski (2007), a região do Vale do Caí possui índices, como PIB, IDH e IDESE, muito próximos aos índices do Rio Grande do Sul, que, por sua vez, é um dos mais altos do país. Essas informações reforçam a importância que a região exerce sobre a economia do estado. A boa expectativa de vida acentua a qualidade de vida da região, porém as altas taxas de analfabetismo corroboram as carências conhecidas em regiões rurais, que demandam investimentos urgentes no setor da educação. Neste sentido, destaca-se a importância de atividades voltadas à compreensão dos processos de gestão da propriedade, de incentivo ao registro de informações e de aprimoramento e de qualificação da tomada de decisões.

De acordo com o IBGE o Município de Montenegro possui uma área de 424,012 km², povoado por 19.580 habitantes destes 29.245 são homens e 30.170 mulheres. A população alfabetizada é de 52.672 pessoas. O PIB per capita a preços correntes no ano de 2011 era de $\mathrm{R} \$ 2$ 29.273,21. O Índice de Desenvolvimento Humanos (IDH) em 2010 chegou a atingir 0,755. Nas áreas rurais o rendimento nominal mediano mensal per capita por domicílios particulares é de $\mathrm{R} \$ 510,00$ já na zona urbana é de $\mathrm{R}$ \$660,00. Já o rendimento nominal médio mensal com rendimento domiciliar, por situação do domicílio rural é de R $\$ 1.609,00$. 
No que se refere ao perfil dos agricultores componentes do Projeto Piloto, no tocante a escolaridade e a idade, destaca-se que 7 possuem ensino médio completo, 2 ensino fundamental completo e 1 ensino fundamental incompleto. A idade média de idade dos entrevistados é de 33,2 anos, sendo 4 estão na faixa de 20 a 30 anos, 4 entre 30 a 40 anos e 2 superior a 40 anos de idade.

\section{Figura 1 - Localização do Vale do Caí e de Montenegro-RS}

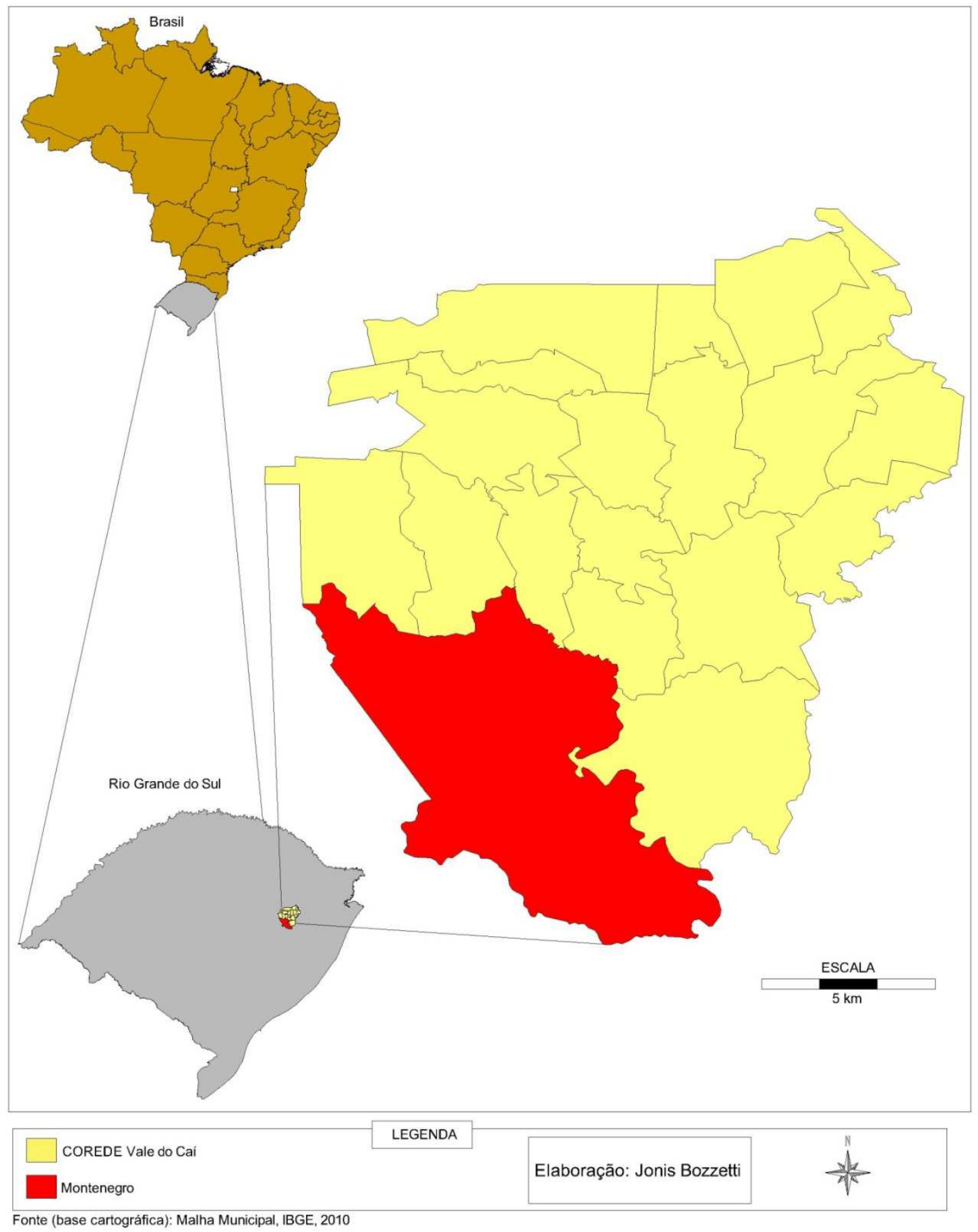


A média de componentes por família é de 4,2 pessoas por propriedade, sendo 4 das propriedades com 5 pessoas, 2 com 4 pessoas e 3 com 3 pessoas. Dos 10 agricultores que compõem o projeto, 9 são do sexo masculino e lé do sexo feminino, sendo que 6 são casados e 4 são solteiros. Em uma relação entre idade e estado civil os casados possuem acima de 30 anos.

Os agricultores foram divididos em duas áreas: Costa da Serra e Campo do meio (Lajeadinho, Santos Reis e Vapor velho) sendo que 3 residem em Costa da Serra e 7 em Campo do Meio.

Quanto à origem étnica dos agricultores 10 afirmam ser de origem alemã, sendo que 2, dentre os 10, também afirmam ter origem portuguesa. Quanto à religião 9 agricultores são evangélicos luteranos e apenas lé católico. Todos os entrevistados afirmam que a família e os amigos como instituições mais confiáveis. Todos os entrevistados possuem automóveis, sendo que 3 destes possuem mais de um veículo na família por propriedade.

Os dados referentes às propriedades rurais destacam-se: a renda média familiar varia entre 1 a 10 salários mínimos, sendo que 3 dizem receber entre 1 a 2 salários mínimos, 1 entre 2 a 3, 3 entre 3 a 5, 1 entre 5 a 10, e 1 superior a 10 salários mínimos.

O tamanho das propriedades fica em média de 32,2 hectares (com propriedades que vão de 21 hectares até 47 hectares), sendo distribuídas entre vários cultivos diferentes, a pesquisa mostrou que todos os agricultores cultivam citricultura na propriedade, 2 criam gado de corte, 7 cultivam silvicultura (eucalipto e acácia), 3 possuem gado leiteiro, 2 possuem suinocultura, 1trabalha com avicultura, 1 cultiva mandioca. A maior parte de área da propriedade é usada para Citricultura.

De acordo com os resultados obtidos, no que se refere ao acesso e ao uso de TICs observou-se que todos os entrevistados possuem celular, 4 possuem computador (tipo PC) em casa e 9 possuem computador (tipo notebook). Destes entrevistados 6 deles utilizam o computador diariamente e 6 também utilizam a internet diariamente, 4 utilizam o computador às vezes e 2 utilizam a internet às vezes. Este acesso à internet ocorre via computador em casa com banda larga (1 entrevistado), por 3G (4 entrevistados), via rádio (4 entrevistados) e não tem acesso internet (1 entrevistado). Quando conectados, 50\% dos entrevistados permanecem por pelo menos uma hora e $50 \%$ entre uma e duas horas.

No que se refere às atividades de extensão, destaca-se que, no momento atual, a equipe realiza o acompanhamento dos agricultores em suas propriedades, buscando motivar o registro das informações necessárias para introdução das informações na planilha eletrônica (Excel) para gestão destas e para qualificação do processo de tomada de decisão. Com base 
em observação realizada pela equipe do projeto, os agricultores apresentam-se receosos e desconfiados quanto ao real retorno relativo ao registro das informações, apresentam dificuldades de compartilhar informações da propriedade com os demais integrantes do grupo e resistência ao desenvolvimento do hábito de registro. É imperativo que os agricultores adotem processos de aprendizagem relacionados com atividades pouco usuais nos sistemas tradicionais de produção, como por exemplo, o hábito do registro das informações e a acompanhamento das despesas e das receitas.

O principal problema não se encontra nas técnicas agropecuárias que, dentro da realidade de cada agricultor, estão plenamente disponíveis. Segundo MARION e SEGATTI (2006), ele reside, sobretudo, na compreensão do funcionamento dos mercados, que impõe articulação com os segmentos pré e pós-porteira, novas formas de negociação e práticas de gestão do processo produtivo. Um número significativo de pequenos agricultores desconhece a evolução do mercado e as alterações nos hábitos de consumo, olhando apenas para a sua atividade como se ela estivesse desvinculada dos demais segmentos produtivos. $\mathrm{O}$ uso de métodos de gestão nas propriedades familiares favorece a inserção ao mercado e a geração de renda nas famílias.

\section{CONSIDERAÇÕES FINAIS}

A introdução e a utilização de TICs no meio rural facilitam a comunicação, a troca de informações e a ampliação do conhecimento dos agricultores, sendo que este último poderá promover o alargamento de oportunidades econômicas, sociais e políticas. Assim, a inclusão digital do campo poderá alavancar os processos de desenvolvimento rural e regional.

O envolvimento da família na introdução das TICs, através de processos de gestão da propriedade (utilização de planilhas de gerenciamento da produção) e da capacitação nas principais tecnologias de informação e de comunicação, tais como o uso da internet, e-mail, acesso a sites e comunicadores instantâneos, possibilitará maior integração, formação de redes de reciprocidade e, acompanhamento e monitoramento das atividades produtivas.

Contudo, destaca-se que a maioria dos softwares de controle da produção e de gestão rural ofertada pelo mercado está totalmente desvinculada da possibilidade de agricultores familiares realizarem a gestão da propriedade independentemente, pois necessitam da ajuda de experts. A construção conjunta de planilhas de acompanhamento poderá permitir a legitimidade dos agricultores e a autonomia do processo gerencial do estabelecimento. No entanto, o processo é lento e gradual, com alta probabilidade de retrocesso, pois o mercado 
agrícola atual exige capacidade de articulação, de formação de redes e de conhecimento macroeconômico conjuntural, variáveis, muitas vezes, desconhecidas por amplo espectro de agricultores familiares.

Assim, observa-se que, no que tange o Projeto Piloto, os agricultores possuem acesso às TICs. No entanto, quanto ao uso da tecnologia, verificou-se ainda há necessidade de domínio e de apropriação do conhecimento para a utilização mais intensiva das TICs, pois o acesso é superficial não levando à apropriação da tecnologia. Embora alguns agricultores já realizem algum tipo de anotação e de acompanhamento, estes representam um caráter simplista e, na maioria das vezes, desconectado de um processo de controle ou de apoio à tomada de decisão.

Portanto, a introdução das novas tecnologias de informação e de comunicação exige uma construção conjunta do conhecimento, a partir de diálogos, de debate e de negociação, buscando o estabelecimento de uma dinâmica de interface e de aprendizagem social. Nesse sentido, as famílias rurais deverão, além de acessar essas tecnologias (infraestrutura), desfrutar de informações compatíveis com seu modo de vida e serem qualificadas para sua utilização. Dessa forma, vários são os desafios para o uso das TICs, posto que não há uma cultura de registro de informações por parte dos agricultores e existe baixo grau de instrução no campo, inadequação das ferramentas existentes e falta de políticas públicas de incentivo.

\section{REFERÊNCIAS}

BATALHA, M. O.; BUAINAIN, A. M.; SOUZA FILHO, H. M. Tecnologia de gestão e agricultura familiar. In: SOUZA FILHO, H. M.; BATALHA, M. O. Gestão integrada da agricultura familiar. São Paulo: edUFSCar, 2004, p. 43-65.

BORDENAVE, J. E. D. de. La información agrícola a La comunicación para el cambio social. In: CIMADEVILLA, G.; THORNTON, R. (editores). A extensão rural em debate Concepções, retrospectivas, mudanças e estratégias para o Mercosul. Buenos Aires: INTA, 2003.

BUAINAIN, A. M., SOUZA FILHO, H.; SILVEIRA, J. M. Inovação tecnológica na agricultura e agricultura familiar. In: LIMA, D; WILKINSON, J.(Org.). Inovação nas tradições da agricultura familiar. Brasília: CNPq/Paralelo, 2007

FEE (Fundação de Economia e Estatística) - Corede Vale do Caí. Rio Grande do Sul: 2010. Disponível em: 〈http://www.fee.tche.br/>. Acesso em: 20 de out. de 2013. 
LOURENZANI, W. L.; PINTO, L. de B.; CARVALHO, E. C. A. de; CARMO, S. M. do. A qualificação em gestão da agricultura familiar: a experiência da Alta Paulista. In: Revista de Ciências e Extensão, v. 4, n. 1, p. 62, 2008.

IBGE. Cidades. Disponível em: 〈http://cod.ibge.gov.br/DBZF〉. Acesso em: 25 de abr. de 2014.

MARION, J. C.; SEGATTI, S. Sistema de gestão de custos nas pequenas propriedades leiteiras.In: Custos e @ gronegócio on line, v. 2, n. 2, 2006.

MILLARD, J. L.. As tecnologias de informação a serviço do desenvolvimento rural. Cadernos do Observatório, n. 4, 2000.

PLOEG, J. D.; LONG, A.; BANKS, J. Rural development: the state of the art. In: PLOEG, J. D.; LONG, A.; BANKS, J. (Ed.) Living countrysides: rural development processes in Europe the state of the art. Netherlands: Elsevier, 2002.

SCHWARTZ, C. A recepção das Tecnologias de Informação e Comunicação entre os agricultores familiares de Santa Maria. Dissertação (Programa de Mestrado em Extensão Rural), 2007.

SILVEIRA, A. C. M. da. (org.). Divulgação científica e tecnologias de informação e comunicação. Santa Maria: FACOS-UFSM, 2003.

SOUZA, J.; BINKOSWKI, P. As Transformações Dos Sistemas Agrários No Vale Do Caí, Rio Grande Do Sul, Texto de Discussão. PPGDR/UFRGS: Porto Alegre, 2007.

THORNTON, R. (Ed.). "El agricultor, internet y las barreras a su adopción”. In: A extensão rural em debate - concepções, retrospectivas, mudanças e estratégias para o Mercosul. Buenos Aires: INTA, p. 323-345, 2003.

VIEIRO, V.; SILVEIRA, A. M. Apropriação de tecnologias de informação e tecnologias de informação no meio rural brasileiro. Cadernos de Ciência \& Tecnologia, Brasília, v. 28, n. 1, p. 257-277, 2011. 